\title{
Gauge Theories on the Light-Front
}

\author{
Stanley J. Brodsky \\ Stanford Linear Accelerator Center, \\ Stanford University, Stanford, California 94309, USA
}

Received on 10 February, 2003.

\begin{abstract}
The light-front quantization of gauge theories in light-cone gauge provides a frame-independent wavefunction representation of relativistic bound states, simple forms for current matrix elements, explicit unitary, and a trivial vacuum. The light-front Hamiltonian form of QCD provides an alternative to lattice gauge theory for the computation of nonperturbative quantities such as the hadronic spectrum and the corresponding eigenfunctions. In the case of the electroweak theory, spontaneous symmetry breaking is represented by the appearance of zero modes of the Higgs field. Light-front quantization then leads to an elegant ghost-free theory of massive gauge particles, automatically incorporating the Lorentz and 't Hooft conditions, as well as the Goldstone boson equivalence theorem.
\end{abstract}

\section{Introduction}

One of the challenges of relativistic quantum field theory is to compute the wavefunctions of bound states such as the amplitudes which determine the quark and gluon substructure of hadrons in quantum chromodynamics. In lightfront quantization [1], one fixes the initial boundary conditions of a composite system as its constituents are intercepted by a single light-wave evaluated on the hyperplane $x^{+}=t+z / c$. The light-front quantization of QCD provides a frame-independent, quantum-mechanical representation of a hadron at the amplitude level, capable of encoding its multi-quark, hidden-color and gluon momentum, helicity, and flavor correlations in the form of universal process-independent and frame-independent hadron wavefunctions [2]. Remarkably, quantum fluctuations of the vacuum are absent if one uses light-front time to quantize the system, so that matrix elements such as the electromagnetic form factors only depend on the currents of the constituents described by the light-cone wavefunctions. The degrees of freedom associated with vacuum phenomena such as spontaneous symmetry breaking in the Higgs model have their counterpart in light-front $k^{+}=0$ zero modes of the fields.

In Dirac's "Front Form" [1], the generator of light-front time translations is $P^{-}=i \frac{\partial}{\partial \tau}$. Boundary conditions are set on the transverse plane labelled by $x_{\perp}$ and $x^{-}=z-$ ct. Given the Lagrangian of a quantum field theory, $P^{-}$can be constructed as an operator on the Fock basis, the eigenstates of the free theory. Since each particle in the Fock basis is on its mass shell, $k^{-} \equiv k^{0}-k^{3}=\frac{k_{\perp}^{2}+m^{2}}{k^{+}}$, and its energy $k^{0}=\frac{1}{2}\left(k^{+}+k^{-}\right)$is positive, only particles with positive momenta $k^{+} \equiv k^{0}+k^{3} \geq 0$ can occur in the Fock basis. Since the total plus momentum $P^{+}=\sum_{n} k_{n}^{+}$is conserved, the light-cone vacuum cannot have any particle content.

The Heisenberg equation on the light-front is

$$
H_{L C}|\Psi\rangle=M^{2}|\Psi\rangle \text {. }
$$

The operator $H_{L C}=P^{+} P^{-}-P_{\perp}^{2}$, the "light-cone Hamiltonian", is frame-independent. This can in principle be solved by diagonalizing the matrix $\left\langle n\left|H_{L C}\right| m\right\rangle$ on the free Fock basis: [2]

$$
\sum_{m}\left\langle n\left|H_{L C}\right| m\right\rangle\langle m \mid \psi\rangle=M^{2}\langle n \mid \Psi\rangle
$$

The eigenvalues $\left\{M^{2}\right\}$ of $H_{L C}=H_{L C}^{0}+V_{L C}$ give the squared invariant masses of the bound and continuum spectrum of the theory. The light-front Fock space is the eigenstates of the free light-front Hamiltonian; i.e., it is a Hilbert space of non-interacting quarks and gluons, each of which satisfy $k^{2}=m^{2}$ and $k^{-}=\frac{m^{2}+k_{\perp}^{2}}{k^{+}} \geq 0$. The projections $\{\langle n \mid \Psi\rangle\}$ of the eigensolution on the $n$-particle Fock states provide the light-front wavefunctions. Thus solving a quantum field theory is equivalent to solving a coupled manybody quantum mechanical problem:

$$
\left[M^{2}-\sum_{i=1}^{n} \frac{m^{2}+k_{\perp}^{2}}{x_{i}}\right] \psi_{n}=\sum_{n^{\prime}} \int\left\langle n\left|V_{L C}\right| n^{\prime}\right\rangle \psi_{n^{\prime}}
$$

where the convolution and sum is understood over the Fock number, transverse momenta, plus momenta, and helicity of the intermediate states. Light-front wavefunctions are also related to momentum-space Bethe-Salpeter wavefunctions by integrating over the relative momenta $k^{-}=k^{0}-k^{z}$ since this projects out the dynamics at $x^{+}=0$.

The light-front quantization of gauge theory can be most conveniently carried out in the light-cone gauge $A^{+}=$ $A^{0}+A^{z}=0$. In this gauge the $A^{-}$field becomes a dependent degree of freedom, and it can be eliminated from the Hamiltonian in favor of a set of specific instantaneous lightfront time interactions. In fact in $Q C D(1+1)$ theory, this instantaneous interaction provides the confining linear $x^{-}$ interaction between quarks. In $3+1$ dimensions, the transverse field $A^{\perp}$ propagates massless spin-one gluon quanta 
with polarization vectors [3] which satisfy both the gauge condition $\epsilon_{\lambda}^{+}=0$ and the Lorentz condition $k \cdot \epsilon=0$.

LF quantization is especially useful for quantum chromodynamics, since it provides a rigorous extension of many-body quantum mechanics to relativistic bound states: the quark, and gluon momenta and spin correlations of a hadron become encoded in the form of universal processindependent, Lorentz-invariant wavefunctions [4]. For example, the eigensolution of a meson in QCD, projected on the eigenstates $\{|n\rangle\}$ of the free Hamiltonian $H_{L C}^{Q C D}(g=$ 0 ) at fixed $\tau=t-z / c$ with the same global quantum numbers, has the expansion:

$$
\begin{aligned}
& \left|\Psi_{M} ; P^{+}, \vec{P}_{\perp}, \lambda\right\rangle= \\
& \sum_{n \geq 2, \lambda_{i}} \int \Pi_{i=1}^{n} \frac{d^{2} k_{\perp i} d x_{i}}{\sqrt{x_{i}} 16 \pi^{3}} \\
& \times 16 \pi^{3} \delta\left(1-\sum_{j}^{n} x_{j}\right) \delta^{(2)}\left(\sum_{\ell}^{n} \vec{k}_{\perp \ell}\right) \\
& \times\left|n ; x_{i} P^{+}, x_{i} \vec{P}_{\perp}+\vec{k}_{\perp i}, \lambda_{i}\right\rangle \psi_{n / M}\left(x_{i}, \vec{k}_{\perp i}, \lambda_{i}\right) .
\end{aligned}
$$

The set of light-front Fock state wavefunctions $\left\{\psi_{n / M}\right\}$ represent the ensemble of quark and gluon states possible when the meson is intercepted at the light-front. The light-front momentum fractions $x_{i}=k_{i}^{+} / P_{\pi}^{+}=\left(k^{0}+k_{i}^{z}\right) /\left(P^{0}+P^{z}\right)$ with $\sum_{i=1}^{n} x_{i}=1$ and $\vec{k}_{\perp i}$ with $\sum_{i=1}^{n} \vec{k}_{\perp i}=\overrightarrow{0}_{\perp}$ represent the relative momentum coordinates of the QCD constituents and are independent of the total momentum of the state.

Remarkably, the scalar light-front wavefunctions $\psi_{n / p}\left(x_{i}, \vec{k}_{\perp i}, \lambda_{i}\right)$ are independent of the proton's momentum $P^{+}=P^{0}+P^{z}$, and $P_{\perp}$. (The light-cone spinors and polarization vectors multiplying $\psi_{n / p}$ are functions of the absolute coordinates.) Thus once one has solved for the light-front wavefunctions, one can compute hadron matrix elements of currents between hadronic states of arbitrary momentum. The actual physical transverse momenta are $\vec{p}_{\perp i}=x_{i} \vec{P}_{\perp}+\vec{k}_{\perp i}$. The $\lambda_{i}$ label the light-front spin $S^{z}$ projections of the quarks and gluons along the quantization $z$ direction. The spinors of the light-front formalism automatically incorporate the Melosh-Wigner rotation. The physical gluon polarization vectors $\epsilon^{\mu}(k, \lambda= \pm 1)$ are specified in light-cone gauge by the conditions $k \cdot \epsilon=0, \eta \cdot \epsilon=\epsilon^{+}=0$. The parton degrees of freedom are thus all physical; there are no ghost or negative metric states.

\section{Properties of Light-Front Wave- functions}

An important feature of the light-front formalism is that the projection $J_{z}$ of the total is kinematical and conserved. Each light-front Fock state component thus satisfies the angular momentum sum rule: $J^{z}=\sum_{i=1}^{n} S_{i}^{z}+\sum_{j=1}^{n-1} l_{j}^{z}$. The summation over $S_{i}^{z}$ represents the contribution of the intrinsic spins of the $n$ Fock state constituents. The summation over orbital angular momenta

$$
l_{j}^{z}=-\mathrm{i}\left(k_{j}^{1} \frac{\partial}{\partial k_{j}^{2}}-k_{j}^{2} \frac{\partial}{\partial k_{j}^{1}}\right)
$$

derives from the $n-1$ relative momenta. This excludes the contribution to the orbital angular momentum due to the motion of the center of mass, which is not an intrinsic property of the hadron. The numerator structure of the lightfront wavefunctions is in large part determined by the angular momentum constraints. Thus wavefunctions generated by perturbation theory provide a template for the numerator structure of nonperturbative light-front wavefunctions.

Dae Sung Hwang, Bo-Qiang Ma, Ivan Schmidt, and I [5] have shown that the light-front wavefunctions generated by the radiative corrections to the electron in QED provide a simple system for understanding the spin and angular momentum decomposition of relativistic systems. This perturbative model also illustrates the interconnections between Fock states of different particle number. The model is patterned after the quantum structure which occurs in the oneloop Schwinger $\alpha / 2 \pi$ correction to the electron magnetic moment [6]. In effect, we can represent a spin- $\frac{1}{2} \quad$ system as a composite of a spin- $\frac{1}{2}$ fermion and spin-one vector boson. A similar model has been used to illustrate the matrix elements and evolution of light-front helicity and orbital angular momentum operators [7]. This representation of a composite system is particularly useful because it is based on two constituents but yet is totally relativistic. We can then explicitly compute the form factors $F_{1}\left(q^{2}\right)$ and $F_{2}\left(q^{2}\right)$ of the electromagnetic current and the various contributions to the form factors $A\left(q^{2}\right)$ and $B\left(q^{2}\right)$ of the energy-momentum tensor.

Recently Ji, Ma, and Yuan [8] have derived perturbative QCD counting rules for light-front wavefunctions with general values of orbital angular momentum which constrain their form at large transverse momentum.

\section{Applications of Light-Front Wave- functions}

Matrix elements of spacelike currents such as spacelike electromagnetic form factors have an exact representation in terms of simple overlaps of the light-front wavefunctions in momentum space with the same $x_{i}$ and unchanged parton number $n[9,10,6]$. The Pauli form factor and anomalous moment are spin-flip matrix elements of $j^{+}$and thus connect states with $\Delta L_{z}=1$. Thus, these quantities are nonzero only if there is nonzero orbital angular momentum of the quarks in the proton. The Dirac form factor is diagonal in $L_{z}$ and is typically dominated at high $Q^{2}$ by highest states with the highest orbital angular momentum.

The formulas for electroweak current matrix elements of $j^{+}$can be easily extended to the $T^{++}$coupling of gravitons. In, fact, one can show that the anomalous gravitomagnetic moment $B(0)$, analogous to $F_{2}(0)$ in electromagnetic current interactions, vanishes identically for any 
system, composite or elementary [5]. This important feature, which follows in general from the equivalence principle $[11,12,13,14,15]$, is obeyed explicitly in the light-front formalism [5].

The light-front Fock representation is specially advantageous in the study of exclusive $B$ decays. For example, we can write down an exact frame-independent representation of decay matrix elements such as $B \rightarrow D \ell \bar{\nu}$ from the overlap of $n^{\prime}=n$ parton conserving wavefunctions and the overlap of $n^{\prime}=n-2$ from the annihilation of a quarkantiquark pair in the initial wavefunction [16]. The offdiagonal $n+1 \rightarrow n-1$ contributions give a new perspective for the physics of $B$-decays. A semileptonic decay involves not only matrix elements where a quark changes flavor, but also a contribution where the leptonic pair is created from the annihilation of a $q \overline{q^{\prime}}$ pair within the Fock states of the initial $B$ wavefunction. The semileptonic decay thus can occur from the annihilation of a nonvalence quark-antiquark pair in the initial hadron.

The "handbag" contribution to the leading-twist offforward parton distributions measured in deeply virtual Compton scattering has a similar light-front wavefunction representation as overlap integrals of light-front wavefunctions $[17,18]$.

The distribution amplitudes $\phi\left(x_{i}, Q\right)$ which appear in factorization formulae for hard exclusive processes are the valence LF Fock wavefunctions integrated over the relative transverse momenta up to the resolution scale $Q$ [3]. These quantities specify how a hadron shares its longitudinal momentum among its valence quarks; they control virtually all exclusive processes involving a hard scale $Q$, including form factors, Compton scattering and photoproduction at large momentum transfer, as well as the decay of a heavy hadron into specific final states [19, 20].

The quark and gluon probability distributions $q_{i}(x, Q)$ and $g(x, Q)$ of a hadron can be computed from the absolute squares of the light-front wavefunctions, integrated over the transverse momentum. All helicity distributions are thus encoded in terms of the light-front wavefunctions. The DGLAP evolution of the structure functions can be derived from the high $k_{\perp}$ properties of the light-front wavefunctions. Thus given the light-front wavefunctions, one can compute [3] all of the leading twist helicity and transversity distributions measured in polarized deep inelastic lepton scattering. Similarly, the transversity distributions and off-diagonal helicity convolutions are defined as a density matrix of the light-front wavefunctions.

However, it is not true that the leading-twist structure functions $F_{i}\left(x, Q^{2}\right)$ measured in deep inelastic lepton scattering are identical to the quark and gluon distributions. For example, it is usually assumed, following the parton model, that the $F_{2}$ structure function measured in neutral current deep inelastic lepton scattering is at leading order in $1 / Q^{2}$ simply $F_{2}\left(x, Q^{2}\right)=\sum_{q} e_{q}^{2} x q\left(x, Q^{2}\right)$, where $x=x_{b j}=Q^{2} / 2 p \cdot q$ and $q(x, Q)$ can be computed from the absolute square of the proton's light-front wavefunction. Recent work by Hoyer, Marchal, Peigne, Sannino, and myself shows that this standard identification is wrong [21]. Gluon exchange between the fast, outgoing partons and the target spectators, which is usually assumed to be an irrelevant gauge artifact, actually affects the leading-twist structure functions in a profound way. The diffractive scattering of the fast outgoing quarks on spectators in the target in turn causes shadowing in the DIS cross section. Thus the depletion of the nuclear structure functions is not intrinsic to the wave function of the nucleus, but is a coherent effect arising from the destructive interference of diffractive channels induced by final-state interactions. Thus the shadowing corrections related to the Gribov-Glauber mechanism, the interference effects of leading twist diffractive processes in nuclei are separate effects in deep inelastic scattering, are not computable from the bound state wavefunctions of the target nucleon or nucleus. Similarly, the effective pomeron distribution of a hadron is not derived from its light-front wavefunction and thus is not a universal property.

Measurements from the HERMES and SMC collaborations show a remarkably large single-spin asymmetry in semi-inclusive pion leptoproduction $\gamma^{*}(q) p \rightarrow \pi X$ when the proton is polarized normal to the photon-to-pion production plane. Recently, Hwang, Schmidt, and I [22] have shown that final-state interactions from gluon exchange between the outgoing quark and the target spectator system lead to single-spin asymmetries in deep inelastic leptonproton scattering at leading twist in perturbative QCD; i.e., the rescattering corrections are not power-law suppressed at large photon virtuality $Q^{2}$ at fixed $x_{b j}$. The existence of such single-spin asymmetries requires a phase difference between two amplitudes coupling the proton target with $J_{p}^{z}= \pm \frac{1}{2}$ to the same final-state, the same amplitudes which are necessary to produce a nonzero proton anomalous magnetic moment. The single-spin asymmetry which arises from such final-state interactions does not factorize into a product of distribution function and fragmentation function, and it is not related to the transversity distribution $\delta q(x, Q)$ which correlates transversely polarized quarks with the spin of the transversely polarized target nucleon. These effects highlight the unexpected importance of final- and initial-state interactions in QCD observables-they lead to leading-twist single-spin asymmetries, diffraction, and nuclear shadowing, phenomena not included in the wavefunction of the target.

\section{Measurements of Light-Front Wavefunctions}

It is possible to measure the light-front wavefunctions of a relativistic hadron by diffractively dissociating it into jets in high-energy hadron-nucleus collisions such as $\pi A \rightarrow$ jetjet $A^{\prime}$. Only the configurations of the incident hadron which have small transverse size and minimal color dipole moments can traverse the nucleus with minimal interactions and leave it intact. The forward diffractive amplitude is thus coherent over the entire nuclear volume and proportional to nuclear number $A$. The fractional momentum distribution of the jets is correlated with the valence quarks' light-cone momentum fractions $x_{i}$. [23, 24, 25, 26]. The QCD mechanisms for hard diffractive dissociation can be more compli- 
cated in the case of proton targets. A review and references is given in Ref. [27].

The fact that Fock states of a hadron with small particle number and small impact separation have small color dipole moments and weak hadronic interactions is a remarkable manifestation of the gauge structure of QCD. It is the basis for the predictions for "color transparency" in hard quasiexclusive [28, 29] and diffractive reactions [24, 25, 26]. The E791 experiment at FermiLab has verified the nuclear number scaling predictions and have thus provided a remarkable confirmation of this consequence of QCD color transparency [23]. The new EVA spectrometer experiment E850 at Brookhaven has also reported striking effects of color transparency in quasi-elastic proton-proton scattering in nuclei [30].

The CLEO collaboration [31] has verified the scaling and angular predictions for the photon-meson to meson form factor $F_{\gamma \pi^{0}}\left(q^{2}\right)$ which is measured in $\gamma^{*} \gamma \rightarrow \pi^{0}$ reactions. The results are in close agreement with the scaling and normalization of the PQCD predictions [32], provided that the pion distribution amplitude $\phi_{\pi}(x, Q)$ is close to the $x(1-x)$ form, the asymptotic solution to the evolution equation. The pion light-front momentum distribution measured in diffractive dijet production in pion-nucleus collisions by the E791 experiment [23] has a similar form [33]. Data [34] for $\gamma \gamma \rightarrow \pi^{+} \pi^{+}+K^{+} K^{-}$at $W=\sqrt{s}>2.5 \mathrm{GeV}$ are also in agreement with the perturbative QCD predictions. Moreover, the angular distribution shows the expected transition to the predicted QCD form as $W$ is raised. A compilation of the two-photon data has been given by Whalley [35]. Measurements of the reaction $\gamma \gamma \rightarrow \pi^{0} \pi^{0}$ are highly sensitive to the shape of the pion distribution amplitude. The perturbative QCD predictions [32] for this channel contrast strongly with model predictions based on the QCD Compton handbag diagram [36].

\section{Higher Particle-Number Fock States}

The light-front Fock state expansion of a hadron contains fluctuations with an arbitrary number of quark and gluon partons. The higher Fock states of the light hadrons describe the sea quark structure of the deep inelastic structure functions, including "intrinsic" strangeness and charm fluctuations specific to the hadron's structure rather than gluon substructure $[37,38]$. The maximal contribution of an intrinsic heavy quark occurs at $x_{Q} \simeq m_{\perp Q} / \sum_{i} m_{\perp}$ where $m_{\perp}=$ $\sqrt{m^{2}+k_{\perp}^{2}}$; i.e. at large $x_{Q}$, since this minimizes the invariant mass $\mathcal{M}_{n}^{2}$. The measurements of the charm structure function by the EMC experiment are consistent with intrinsic charm at large $x$ in the nucleon with a probability of order $0.6 \pm 0.3 \%$ [39] which is consistent with the recent estimates based on instanton fluctuations [40]. Franz, Polyakov, and Goeke have analyzed the properties of the intrinsic heavyquark fluctuations in hadrons using the operator-product expansion [40]. For example, the light-cone momentum fraction carried by intrinsic heavy quarks in the proton $x_{Q \bar{Q}}$ as measured by the $T^{++}$component of the energy-momentum tensor is related in the heavy-quark limit to the forward matrix element $\left\langle p\left|\operatorname{tr}_{c}\left(G^{+\alpha} G^{+\beta} G_{\alpha \beta}\right) / m_{Q}^{2}\right| p\right\rangle$, where $G^{\mu \nu}$ is the gauge field strength tensor. Diagrammatically, this can be described as a heavy quark loop in the proton selfenergy with four gluons attached to the light, valence quarks. Since the non-Abelian commutator $\left[A_{\alpha}, A_{\beta}\right]$ is involved, the heavy quark pairs in the proton wavefunction are necessarily in a color-octet state. It follows from dimensional analysis that the momentum fraction carried by the $Q \bar{Q}$ pair scales as $k_{\perp}^{2} / m_{Q}^{2}$ where $k_{\perp}$ is the typical momentum in the hadron wave function. In contrast, in the case of Abelian theories, the contribution of an intrinsic, heavy lepton pair to the bound state's structure first appears in $O\left(1 / m_{L}^{4}\right)$.

The presence of intrinsic charm quarks in the $B$ wave function provides new mechanisms for $B$ decays. For example, Chang and Hou have considered the production of final states with three charmed quarks such as $B \rightarrow J / \psi D \pi$ and $B \rightarrow J / \psi D^{*}$ [41]; these final states are difficult to realize in the valence model, yet they occur naturally when the $b$ quark of the intrinsic charm Fock state $|b \bar{u} c \bar{c}\rangle$ decays via $b \rightarrow c \bar{u} d$. Susan Gardner and I have shown that the presence of intrinsic charm in the hadrons' light-front wave functions, even at a few percent level, provides new, competitive decay mechanisms for $B$ decays which are nominally CKM-suppressed [42]. Similarly, Karliner and I [43] have shown that the transition $J / \psi \rightarrow \rho \pi$ can occur by the rearrangement of the $c \bar{c}$ from the $J / \psi$ into the $|q \bar{q} c \bar{c}\rangle$ intrinsic charm Fock state of the $\rho$ or $\pi$. On the other hand, the overlap rearrangement integral in the decay $\psi^{\prime} \rightarrow \rho \pi$ will be suppressed since the intrinsic charm Fock state radial wavefunction of the light hadrons will evidently not have nodes in its radial wavefunction. This observation provides a natural explanation of the long-standing puzzle [44] why the $J / \psi$ decays prominently to two-body pseudoscalar-vector final states, breaking hadron helicity conservation [45], whereas the $\psi^{\prime}$ does not.

\section{Light-Front Quantization of QCD}

Quantum field theories are usually quantized at fixed "instant" time $t$. The resulting Hamiltonian theory is complicated by the dynamical nature of the vacuum state and the fact that relativistic boosts are not kinematical but involve interactions. The calculation of even the simplest current matrix elements requires the computation of amplitudes where the current interacts with particles resulting from the fluctuations of the vacuum. All of these problems are dramatically alleviated when one quantizes quantum field theories at fixed light-cone time $\tau$. A review of the development of light-front quantization of QCD and other quantum field theories is given in Ref. [2].

Prem Srivastava and I [46] have presented a new systematic study of light-front-quantized gauge theory in lightcone gauge using a Dyson-Wick S-matrix expansion based on light-front-time-ordered products. The Dirac bracket method is used to identify the independent field degrees of freedom [47]. In our analysis one imposes the light-cone gauge condition as a linear constraint using a Lagrange multiplier, rather than a quadratic form. We then find that the 
LF-quantized free gauge theory simultaneously satisfies the covariant gauge condition $\partial \cdot A=0$ as an operator condition as well as the LC gauge condition. The gluon propagator has the form

$$
\left\langle 0\left|T\left(A_{\mu}^{a}{ }_{\mu}(x) A^{b}{ }_{\nu}(0)\right)\right| 0\right\rangle=\frac{i \delta^{a b}}{(2 \pi)^{4}} \int d^{4} k e^{-i k \cdot x} \frac{D_{\mu \nu}(k)}{k^{2}+i \epsilon}
$$

where we have defined

$$
D_{\mu \nu}(k)=D_{\nu \mu}(k)=-g_{\mu \nu}+\frac{n_{\mu} k_{\nu}+n_{\nu} k_{\mu}}{(n \cdot k)}-\frac{k^{2}}{(n \cdot k)^{2}} n_{\mu} n_{\nu}
$$

Here $n_{\mu}$ is a null four-vector, gauge direction, whose components are chosen to be $n_{\mu}=\delta_{\mu}{ }^{+}, n^{\mu}=\delta^{\mu}{ }_{-}$. Note also

$$
\begin{aligned}
D_{\mu \lambda}(k) D^{\lambda}{ }_{\nu}(k)=D_{\mu \perp}(k) D^{\perp}{ }_{\nu}(k) & =-D_{\mu \nu}(k), \\
k^{\mu} D_{\mu \nu}(k)=0, n^{\mu} D_{\mu \nu}(k) & \equiv D_{-\nu}(k)=0, \\
D_{\lambda \mu}(q) D^{\mu \nu}(k) D_{\nu \rho}\left(q^{\prime}\right) & =-D_{\lambda \mu}(q) D^{\mu \rho}\left(q^{\prime}\right) .
\end{aligned}
$$

The gauge field propagator $i D_{\mu \nu}(k) /\left(k^{2}+i \epsilon\right)$ is transverse not only to the gauge direction $n_{\mu}$ but also to $k_{\mu}$, i.e., it is doubly-transverse. Thus $D$ represents the polarization sum over physical propagating modes. The last term proportional to $n_{\mu} n_{\nu}$ in the gauge propagator does not appear in the usual formulations of light-cone gauge. However, in tree graph calculations it cancels against instantaneous gluon exchange contributions.

The remarkable properties of (the projector) $D_{\nu \mu}$ greatly simplifies the computations of loop amplitudes. For example, the coupling of gluons to propagators carrying high momenta is automatic. In the case of tree graphs, the term proportional to $n_{\mu} n_{\nu}$ cancels against the instantaneous gluon exchange term. However, in the case of loop diagrams, the separation needs to be maintained so that one can identify the correct one-particle-irreducible contributions. The absence of collinear divergences in irreducible diagrams in the light-cone gauge greatly simplifies the leading-twist factorization of soft and hard gluonic corrections in high momentum transfer inclusive and exclusive reactions [3] since the numerators associated with the gluon coupling only have transverse components.

The interaction Hamiltonian of QCD in light-cone gauge can be derived by systematically applying the Dirac bracket method to identify the independent fields [46]. It contains the usual Dirac interactions between the quarks and gluons, the three-point and four-point gluon non-Abelian interactions plus instantaneous light-front-time gluon exchange and quark exchange contributions

$$
\begin{aligned}
\mathcal{H}_{\text {int }}= & -g \bar{\psi}^{i} \gamma^{\mu} A_{\mu}{ }^{i j} \psi^{j} \\
& +\frac{g}{2} f^{a b c}\left(\partial_{\mu} A^{a}{ }_{\nu}-\partial_{\nu} A^{a}{ }_{\mu}\right) A^{b \mu} A^{c \nu} \\
& +\frac{g^{2}}{4} f^{a b c} f^{a d e} A_{b \mu} A^{d \mu} A_{c \nu} A^{e \nu} \\
& -\frac{g^{2}}{2} \bar{\psi}^{i} \gamma^{+}\left(\gamma^{\perp^{\prime}} A_{\perp^{\prime}}\right)^{i j} \frac{1}{i \partial_{-}}\left(\gamma^{\perp} A_{\perp}\right)^{j k} \psi^{k} \\
& -\frac{g^{2}}{2} j^{+}{ }_{a} \frac{1}{\left(\partial_{-}\right)^{2}} j^{+}{ }_{a}
\end{aligned}
$$

where

$$
j^{+}{ }_{a}=\bar{\psi}^{i} \gamma^{+}\left(t_{a}\right)^{i j} \psi^{j}+f_{a b c}\left(\partial_{-} A_{b \mu}\right) A^{c \mu} .
$$

The renormalization constants in the non-Abelian theory have been shown [46] to satisfy the identity $Z_{1}=Z_{3}$ at oneloop order, as expected in a theory with only physical gauge degrees of freedom. The renormalization factors in the light-cone gauge are independent of the reference direction $n^{\mu}$. The QCD $\beta$ function computed in the noncovariant LC gauge agrees with the conventional theory result $[48,49]$. Dimensional regularization and the Mandelstam-Leibbrandt prescription $[51,50,52]$ for LC gauge were used to define the Feynman loop integrations [53]. There are no FaddeevPopov or Gupta-Bleuler ghost terms.

The running coupling constant and QCD $\beta$ function have also been computed at one loop in the doubly-transverse light-cone gauge [46]. It is also possible to effectively quantize QCD using light-front methods in covariant Feynman gauge [54].

It is well-known that the light-cone gauge itself is not completely defined until one specifies a prescription for the poles of the gauge propagator at $n \cdot k=0$. The Mandelstam-Liebbrandt prescription has the advantage of preserving causality and analyticity, as well as leading to proofs of the renormalizability and unitarity of Yang-Mills theories [55]. The ghosts which appear in association with the Mandelstam-Liebbrandt prescription from the single poles have vanishing residue in absorptive parts, and thus do not disturb the unitarity of the theory.

A remarkable advantage of light-front quantization is that the vacuum state $|0\rangle$ of the full QCD Hamiltonian evidently coincides with the free vacuum. The light-front vacuum is effectively trivial if the interaction Hamiltonian applied to the perturbative vacuum is zero. Note that all particles in the Hilbert space have positive energy $k^{0}=$ $\frac{1}{2}\left(k^{+}+k^{-}\right)$, and thus positive light-front $k^{ \pm}$. Since the plus momenta $\sum k_{i}^{+}$is conserved by the interactions, the perturbative vacuum can only couple to states with particles in which all $k_{i}^{+}=0$; i.e., so called zero-mode states. Bassetto and collaborators [56] have shown that the computation of the spectrum of $Q C D(1+1)$ in equal time quantization requires constructing the full spectrum of non perturbative contributions (instantons). In contrast, in the light-front quantization of gauge theory, where the $k^{+}=0$ singularity of the instantaneous interaction is defined by a simple infrared regularization, one obtains the correct spectrum of $Q C D(1+1)$ without any need for vacuum-related contributions.

Zero modes of auxiliary fields are necessary to distinguish the theta-vacua of massless $\operatorname{QED}(1+1)[57,58,59]$, or to represent a theory in the presence of static external boundary conditions or other constraints. Zero-modes provide the light-front representation of spontaneous symmetry breaking in scalar theories [60]. 


\section{Light-Front Quantization of the Standard Model}

Prem Srivastava and I have also shown how light-front quantization can be applied to the Glashow, Weinberg and Salam (GWS) model of electroweak interactions based on the nonabelian gauge group $S U(2)_{W} \times U(1)_{Y}$ [61]. This theory contains a nonabelian Higgs sector which triggers spontaneous symmetry breaking (SSB). A convenient way of implementing SSB and the (tree level) Higgs mechanism in the front form theory was developed earlier by Srivastava $[62,63,64]$. One separates the quantum fluctuation fields from the corresponding dynamical bosonic condensate (or zero-longitudinal-momentum-mode) variables, before applying the Dirac procedure in order to construct the Hamiltonian formulation. The canonical quantization of LC gauge GWS electroweak theory in the front form can be derived by using the Dirac procedure to construct a selfconsistent LF Hamiltonian theory. This leads to an attractive new formulation of the Standard Model of the strong and electroweak interactions which does not break the physical vacuum and has well-controlled ultraviolet behavior. The only ghosts which appear in the formalism are the $n \cdot k=0$ modes of the gauge field associated with regulating the lightcone gauge prescription. The massive gauge field propagator has good asymptotic behavior in accordance with a renormalizable theory, and the massive would-be Goldstone fields can be taken as physical degrees of freedom.

For example, consider the Abelian Higgs model. The interaction Lagrangian is

$$
\mathcal{L}=-\frac{1}{4} F_{\mu \nu} F^{\mu \nu}+\left|D_{\mu} \phi\right|^{2}-V\left(\phi^{\dagger} \phi\right)
$$

where

$$
D_{\mu}=\partial_{\mu}+i e A_{\mu}
$$

and

$$
V(\phi)=\mu^{2} \phi^{\dagger} \phi+\lambda\left(\phi^{\dagger} \phi\right)^{2},
$$

with $\mu^{2}<0, \lambda>0$. The complex scalar field $\phi$ is decomposed as

$$
\phi(x)=\frac{1}{\sqrt{2}} v+\varphi(x)=\frac{1}{\sqrt{2}}[v+h(x)+i \eta(x)]
$$

where $v$ is the $k^{+}=0$ zero mode determined by the minimum of the potential: $v^{2}=-\frac{\mu^{2}}{\lambda}, h(x)$ is the dynamical Higgs field, and $\eta(x)$ is the Nambu-Goldstone field. The quantization procedure determines $\partial \cdot A=M R$, the 't Hooft condition. One can now eliminate the zero mode component of the Higgs field $v$ which gives masses for the fundamental quantized fields. The $A_{\perp}$ field then has mass $M=e v$ and the Higgs field acquires mass $m_{h}^{2}=2 \lambda v^{2}=-2 \mu^{2}$.

A new aspect of LF quantization, is that the third polarization of the quantized massive vector field $A^{\mu}$ with four momentum $k^{\mu}$ has the form $E_{\mu}^{(3)}=n_{\mu} M / n \cdot k$. Since $n^{2}=0$, this non-transverse polarization vector has zero norm. However, when one includes the constrained interactions of the Goldstone particle, the effective longitudinal polarization vector of a produced vector particle is
$E_{\text {eff } \mu}^{(3)}=E_{\mu}^{(3)}-k_{\mu} k \cdot E^{(3)} / k^{2}$ which is identical to the usual polarization vector of a massive vector with norm $E_{\text {eff }}^{(3)} \cdot E_{\text {eff }}^{(3)}=-1$. Thus, unlike the conventional quantization of the Standard Model, the Goldstone particle only provides part of the physical longitudinal mode of the electroweak particles.

In the LC gauge LF framework, the free massive gauge fields in the electroweak theory satisfy simultaneously the 't Hooft conditions as an operator equation. The sum over the three physical polarizations is given by $K_{\mu \nu}$

$$
\begin{aligned}
K_{\mu \nu}(k) & =\sum_{(\alpha)} E_{\mu}^{(\alpha)} E_{\nu}^{(\alpha)}=D_{\mu \nu}(k)+\frac{M^{2}}{\left(k^{+}\right)^{2}} n_{\mu} n(15) \\
& =-g_{\mu \nu}+\frac{n_{\mu} k_{\nu}+n_{\nu} k_{\mu}}{(n \cdot k)}-\frac{\left(k^{2}-M^{2}\right)}{(n \cdot k)^{2}} n_{\mu} n_{\nu}
\end{aligned}
$$

which satisfies: $k^{\mu} K_{\mu \nu}(k)=\left(M^{2} / k^{+}\right) n_{\nu}$ and $k^{\mu} k^{\nu} K_{\mu \nu}(k)=M^{2}$. The free propagator of the massive gauge field $A_{\mu}$ is

$$
\begin{aligned}
&\left\langle 0\left|T\left(A_{\mu}(x) A_{\nu}(y)\right)\right| 0\right\rangle= \\
& \frac{i}{(2 \pi)^{4}} \int d^{4} k \frac{K_{\mu \nu}(k)}{\left(k^{2}-M^{2}+i \epsilon\right)} e^{-i k \cdot(x-y)} .
\end{aligned}
$$

It does not have the bad high energy behavior found in the (Proca) propagator in the unitary gauge formulation, where the would-be Nambu-Goldstone boson is gauged away.

In the limit of vanishing mass of the vector boson, the gauge field propagator goes over to the doubly transverse gauge, $\left(n^{\mu} D_{\mu \nu}(k)=k^{\mu} D_{\mu \nu}(k)=0\right)$, the propagator found [46] in QCD. The numerator of the gauge propagator $K_{\mu \nu}(k)$ also has important simplifying properties, similar to the ones associated with the projector $D_{\mu \nu}(k)$. The transverse polarization vectors for massive or massless vector boson may be taken to be $E_{(\perp)}^{\mu}(k) \equiv-D_{\perp}^{\mu}(k)$, whereas the non-transverse third one in the massive case is found to be parallel to the LC gauge direction $E_{\mu}^{(3)}(k)=$ $-\left(M / k^{+}\right) n_{\mu}$. Its projection along the direction transverse to $k_{\mu}$ shares the spacelike vector property carried by $E_{(\perp)}^{\mu}(k)$. The Goldstone boson or electroweak equivalence theorem becomes transparent in the LF formulation.

The interaction Hamiltonian for the Abelian Higgs model in LC gauge $A^{+}=0$, is found to be

$$
\begin{aligned}
& -\mathcal{H}_{\text {int }}=\mathcal{L}_{\text {int }} \\
& =e M A_{\mu} A^{\mu} h-\frac{e m_{h}^{2}}{2 M}\left(\eta^{2}+h^{2}\right) h \\
& +e\left(h \partial_{\mu} \eta-\eta \partial_{\mu} h\right) A^{\mu}+\frac{e^{2}}{2}\left(h^{2}+\eta^{2}\right) A_{\mu} A^{\mu} \\
& -\frac{\lambda}{4}\left(\eta^{2}+h^{2}\right)^{2}-\frac{e^{2}}{2}\left(\frac{1}{\partial_{-}} j^{+}\right)\left(\frac{1}{\partial_{-}} j^{+}\right)
\end{aligned}
$$

where $j_{\mu}=\left(h \partial_{\mu} \eta-\eta \partial_{\mu} h\right)$. The last term here is the additional quartic instantaneous interaction in the LF theory quantized in the LC gauge No new instantaneous cubic interaction terms are introduced. The massive gauge field, when the mass is generated by the Higgs mechanism, is described 
in our LC gauge framework by the independent fields $A_{\perp}$ and $\eta$; the component $A^{-}$is a dependent field.

The interaction Hamiltonian of the Standard Model can be written in a compact form by retaining the dependent components $A^{-}$and $\psi_{-}$in the formulation. Its form closely resembles the interaction Hamiltonian of covariant theory, except for the presence of additional instantaneous fourpoint interactions. The resulting Dyson-Wick perturbation theory expansion based on equal-LF-time ordering has also been constructed, allowing one to perform higher-order computations in a straightforward fashion.

The singularities in the noncovariant pieces of the field propagators may be defined using the causal ML prescription for $1 / k^{+}$when we employ dimensional regularization, as was shown in our earlier work on QCD. The powercounting rules in LC gauge then become similar to those found in covariant gauge theory.

Spontaneous symmetry breaking is thus implemented in a novel way when one quantizes the Standard Model at fixed light-front time $\tau=x^{+}$. In the general case, the Higgs field $\phi_{i}(x)$ can be separated into two components:

$$
\phi_{i}\left(\tau, x^{-}, \vec{x}_{\perp}\right)=\omega_{i}\left(\tau, \vec{x}_{\perp}\right)+\varphi\left(\tau, x^{-}, \vec{x}_{\perp}\right),
$$

where $\omega_{i}$ is a classical $k^{+}=0$ zero-mode field and $\varphi$ is the dynamical quantized field. Here $i$ is the weak-isospin index. The zero-mode component is determined by solving the Euler-Lagrange tree-level condition:

$$
V_{i}^{\prime}(\omega)-\partial_{\perp} \partial_{\perp} \omega_{i}=0 .
$$

A nonzero value for $\omega_{i}$ corresponds to spontaneous symmetry breaking. The nonzero $\omega_{i}$ couples to the gauge boson and Fermi fields through the Yukawa interactions of the Standard Model. It can then be eliminated from the theory in favor of mass terms for the fundamental matter fields in the effective theory. The resulting masses are identical to those of the usual Higgs implementation of spontaneous symmetry breaking in the Standard Model.

The generators of isospin rotations are defined from the dynamical Higgs fields:

$$
G_{a}=-i \int d x^{\perp} d x^{-}\left(\partial_{-} \varphi\right)_{i}\left(t_{a}\right)_{i j} \varphi_{j}
$$

Note that the weak-isospin charges and the currents corresponding to $G_{a}$ are not conserved if the zero mode $\omega_{i}$ is nonzero since the cross terms in $\varphi$, and $\omega$ are missing. Thus $\left[H_{L F}, G_{a}\right] \neq 0$. Nevertheless, the charges annihilate the vacuum: $G_{a} \mid 0>_{L F}=0$, since the dynamical fields $\varphi_{i}$ have no support on the LF vacuum, and all quanta have positive $k^{+}$. Thus the LF vacuum remains equal to the perturbative vacuum; it is unaffected by the occurrence of spontaneous symmetry breaking.

In effect one can interpret the $k^{+}=0$ zero mode field $\omega_{i}$ as an $x^{-}$-independent external field, analogous to an applied constant electric or magnetic field in atomic physics. In this interpretation, the zero mode is a remnant of a Higgs field which persists from early cosmology; the LF vacuum however remains unchanged and unbroken.

\section{Discretized Light-Front Quantiza- tion}

If one imposes periodic boundary conditions in $x^{-}=$ $t+z / c$, then the plus momenta become discrete: $k_{i}^{+}=$ $\frac{2 \pi}{L} n_{i}, P^{+}=\frac{2 \pi}{L} K$, where $\sum_{i} n_{i}=K[65,66]$. For a given "harmonic resolution" $K$, there are only a finite number of ways positive integers $n_{i}$ can sum to a positive integer $K$. Thus at a given $K$, the dimension of the resulting light-front Fock state representation of the bound state is rendered finite without violating Lorentz invariance. The eigensolutions of a quantum field theory, both the bound states and continuum solutions, can then be found by numerically diagonalizing a frame-independent light-front Hamiltonian $H_{L C}$ on a finite and discrete momentum-space Fock basis. Solving a quantum field theory at fixed light-front time $\tau$ thus can be formulated as a relativistic extension of Heisenberg's matrix mechanics. The continuum limit is reached for $K \rightarrow \infty$. This formulation of the non-perturbative light-front quantization problem is called "discretized light-cone quantization" (DLCQ) [66]. The method preserves the frameindependence of the Front form.

The DLCQ method has been used extensively for solving one-space and one-time theories [2], including applications to supersymmetric quantum field theories [67] and specific tests of the Maldacena conjecture [68]. There has been progress in systematically developing the computation and renormalization methods needed to make DLCQ viable for QCD in physical spacetime. For example, John Hiller, Gary McCartor, and I $[69,70,71]$ have shown how DLCQ can be used to solve $3+1$ theories despite the large numbers of degrees of freedom needed to enumerate the Fock basis. A key feature of our work is the introduction of Pauli Villars fields to regulate the UV divergences and perform renormalization while preserving the frame-independence of the theory. A recent application of DLCQ to a 3+1 quantum field theory with Yukawa interactions is given in Ref. [69]. One can also define a truncated theory by eliminating the higher Fock states in favor of an effective potential $[73,72,74]$. Spontaneous symmetry breaking and other nonperturbative effects associated with the instant-time vacuum are hidden in dynamical or constrained zero modes on the light-front. An introduction is given in Refs. [75, 57]. A review of DLCQ and its applications is given in Ref. [2].

The pion distribution amplitude has been computed using a combination of the discretized DLCQ method for the $x^{-}$and $x^{+}$light-front coordinates with a spatial lattice $[76,77,78,79]$ in the transverse directions. A finite lattice spacing $a$ can be used by choosing the parameters of the effective theory in a region of renormalization group stability to respect the required gauge, Poincaré, chiral, and continuum symmetries.

Dyson-Schwinger models [80] of hadronic BetheSalpeter wavefunctions can also be used to predict lightfront wavefunctions and hadron distribution amplitudes by integrating over the relative $k^{-}$momentum. 


\section{A Light-Front Event Amplitude Generator}

The light-front formalism can be used as an "event amplitude generator" for high energy physics reactions where each particle's final state is completely labelled in momentum, helicity, and phase. The application of the light-front time evolution operator $P^{-}$to an initial state systematically generates the tree and virtual loop graphs of the $T$-matrix in light-front time-ordered perturbation theory in light-cone gauge. Given the interactions of the light-front interaction Hamiltonian, any amplitude in QCD and the electroweak theory can be computed. For example, this method can be used to automatically compute the tree diagram hard amplitudes $T_{H}$ needed to for computing hard scattering amplitudes such as the deuteron form factor or $p p$ elastic scattering.

At higher orders, loop integrals only involve integrations over the momenta of physical quanta and physical phase space $\prod d^{2} k_{\perp i} d k_{i}^{+}$. Renormalized amplitudes can be explicitly constructed by subtracting from the divergent loops amplitudes with nearly identical integrands corresponding to the contribution of the relevant mass and coupling counter terms (the "alternating denominator method") [81]. The natural renormalization scheme to use for defining the coupling in the event amplitude generator is a physical effective charge such as the pinch scheme [82]. The argument of the coupling is then unambiguous [83]. The DLCQ boundary conditions can be used to discretize the phase space and limit the number of contributing intermediate states without violating Lorentz invariance. Since one avoids dimensional regularization and nonphysical ghost degrees of freedom, this method of generating events at the amplitude level could provide a simple but powerful tool for simulating events both in QCD and the Standard Model. Alternatively, one can construct the $T$-matrix for scattering in QCD using light-front quantization and the event amplitude generator; one can then probe its spectrum by finding zeros of the resolvant.

\section{Acknowledgments}

Work supported by the Department of Energy under contract number DE-AC03-76SF00515. This talk is in large part based on collaborations with the late Professor Prem Srivastava, the outstanding theorist from the Universidade do Estado de Rio de Janeiro and a key innovator of the light-front formalism. I thank Professor Beatriz Gay Ducati and the other organizers of this meeting for their outstanding hospitality in Brazil.

\section{References}

[1] P. A. Dirac, Rev. Mod. Phys. 21, 392 (1949).

[2] For a review and references, see S. J. Brodsky, Acta Phys. Polon. B 32, 4013 (2001) [arXiv:hep-ph/0111340], and S. J. Brodsky, H. C. Pauli, and S. S. Pinsky, Phys. Rept. 301, 299 (1998) [arXiv:hep-ph/9705477].
[3] G. P. Lepage and S. J. Brodsky, Phys. Rev. D 22, 2157 (1980).

[4] See, e.g., S. J. Brodsky, and H. C. Pauli, Light-Cone Quantization and QCD, Lecture Notes in Physics, vol. 396, eds., H. Mitter et al., Springer-Verlag, Berlin, 1991.

[5] S. J. Brodsky, D. S. Hwang, B. Q. Ma, and I. Schmidt, Nucl. Phys. B 593, 311 (2001) [arXiv:hep-th/0003082].

[6] S. J. Brodsky and S. D. Drell, Phys. Rev. D 22, 2236 (1980).

[7] A. Harindranath and R. Kundu, Phys. Rev. D59, 116013 (1999), hep-ph/9802406.

[8] X. d. Ji, J. P. Ma, and F. Yuan, arXiv:hep-ph/0301141.

[9] S. D. Drell and T. Yan, Phys. Rev. Lett. 24, 181 (1970).

[10] G. B. West, Phys. Rev. Lett. 24, 1206 (1970).

[11] L. Okun and I. Yu. Kobzarev, ZhETF, 431904 (1962) (English translation: JETP 161343 (1963)); L. Okun, in proceedings of the International Conference on Elementary Particles, 4th, Heidelberg, Germany (1967). Edited by H. Filthuth. North-Holland, (1968).

[12] X. Ji, hep-ph/9610369.

[13] X. Ji, Phys. Rev. Lett. 78, 610 (1997), hep-ph/9603249.

[14] X. Ji, Phys. Rev. D55, 7114 (1997), hep-ph/9609381.

[15] O. V. Teryaev, hep-ph/9904376. 9904376;

[16] S. J. Brodsky and D. S. Hwang, Nucl. Phys. B 543, 239 (1999) [hep-ph/9806358].

[17] S. J. Brodsky, M. Diehl, and D. S. Hwang, hep-ph/0009254.

[18] M. Diehl, T. Feldmann, R. Jakob, and P. Kroll, hep$\mathrm{ph} / 0009255$.

[19] M. Beneke, G. Buchalla, M. Neubert, and C. T. Sachrajda, Phys. Rev. Lett. 83, 1914 (1999) [hep-ph/9905312].

[20] Y. Keum, H. Li, and A. I. Sanda, hep-ph/0004004.

[21] S. J. Brodsky, P. Hoyer, N. Marchal, S. Peigne, and F. Sannino, Phys. Rev. D 65, 114025 (2002) [arXiv:hep$\mathrm{ph} / 0104291]$.

[22] S. J. Brodsky, D. S. Hwang, and I. Schmidt, deep inelastic scattering," Phys. Lett. B 530, 99 (2002) [arXiv:hep$\mathrm{ph} / 0201296]$.

[23] D. Ashery [E791 Collaboration], hep-ex/9910024.

[24] G. Bertsch, S. J. Brodsky, A. S. Goldhaber, and J. F. Gunion, Phys. Rev. Lett. 47, 297 (1981).

[25] L. Frankfurt, G. A. Miller, and M. Strikman, Phys. Lett. B304, 1 (1993) [hep-ph/9305228].

[26] L. Frankfurt, G. A. Miller, and M. Strikman, Found. Phys. 30, 533 (2000) [hep-ph/9907214].

[27] S. J. Brodsky, arXiv:hep-ph/0208158.

[28] S. J. Brodsky and A. H. Mueller, Phys. Lett. B206, 685 (1988).

[29] L. L. Frankfurt and M. I. Strikman, Phys. Rept. 160, 235 (1988).

[30] A. Leksanov et al., Phys. Rev. Lett. 87, 212301 (2001) [arXiv:hep-ex/0104039].

[31] J. Gronberg et al. [CLEO Collaboration], Phys. Rev. D 57, 33 (1998) [arXiv:hep-ex/9707031].

[32] S. J. Brodsky and G. P. Lepage, Phys. Rev. D 24, 1808 (1981). 
[33] D. Ashery and H. C. Pauli, arXiv:hep-ph/0301113.

[34] H. Aihara et al. [TPC/Two Gamma Collaboration], Phys. Rev. Lett. 57, 404 (1986).

[35] M. R. Whalley, J. Phys. G 27, A1 (2001).

[36] M. Diehl, P. Kroll, and C. Vogt, Phys. Lett. B 532, 99 (2002) [arXiv:hep-ph/0112274].

[37] S. J. Brodsky, P. Hoyer, C. Peterson, and N. Sakai, Phys. Lett. B93, 451 (1980).

[38] S. J. Brodsky, C. Peterson, and N. Sakai, Phys. Rev. D 23, 2745 (1981).

[39] B. W. Harris, J. Smith, and R. Vogt, Nucl. Phys. B461, 181 (1996) [hep-ph/9508403].

[40] M. Franz, M. V. Polyakov, and K. Goeke, Phys. Rev. D 62, 074024 (2000) [hep-ph/0002240].

[41] C. V. Chang and W. Hou, hep-ph/0101162.

[42] S. J. Brodsky and S. Gardner, hep-ph/0108121.

[43] S. J. Brodsky and M. Karliner, Phys. Rev. Lett. 78, 4682 (1997) [hep-ph/9704379].

[44] S. J. Brodsky, G. P. Lepage, and S. F. Tuan, Phys. Rev. Lett. 59, 621 (1987).

[45] S. J. Brodsky and G. P. Lepage, Phys. Rev. D 24, 2848 (1981).

[46] P. P. Srivastava and S. J. Brodsky, Phys. Rev. D 64, 045006 (2001) [arXiv:hep-ph/0011372].

[47] D. M. Ditman and I. V. Tyutin, Quantization of Fields with Constraints, Springer-Verlag, 1990.

[48] D. J. Gross and F. Wilczek, Phys. Rev. Lett. 30, 1343 (1973).

[49] H. D. Politzer, Phys. Rev. Lett. 30, 1346 (1973).

[50] G. Leibbrandt, Rev. Mod. Phys. 59, 1067 (1987).

[51] S. Mandelstam, Nucl. Phys. B 213, 149 (1983).

[52] A. Bassetto, M. Dalbosco, I. Lazzizzera, and R. Soldati, Phys. Rev. D 31, 2012 (1985).

[53] A. Bassetto, Nucl. Phys. Proc. Suppl. 51C, 281 (1996) [arXiv:hep-ph/9605421].

[54] P. P. Srivastava and S. J. Brodsky, Phys. Rev. D61, 025013 (2000), hep-ph/9906423.

[55] A. Bassetto, G. Nardelli, and R. Soldati, Singapore, Singapore: World Scientific (1991) 227 p.

[56] A. Bassetto, L. Griguolo, and F. Vian, hep-th/9911036.

[57] K. Yamawaki, arXiv:hep-th/9802037.

[58] G. McCartor, hep-th/0004139.

[59] P. P. Srivastava, Phys. Lett. B448, 68 (1999) [hepth/9811225].

[60] S. S. Pinsky and B. van de Sande, Phys. Rev. D49, 2001 (1994), hep-ph/9310330.

[61] S. Glashow, Nucl. Phys. 22, 579 (1961); S. Weinberg, Phys. Rev. Lett 19, 1264 (1967); A. Salam, in Elementary Particle Theory, ed. N. Svartholm (Almqvist and Wiksells, Stockholm, 1969), p. 367.
[62] P. P. Srivastava, On spontaneous symmetry breaking mechanism in light-front quantized theory, Ohio-State University preprint, November 1991, CBPF, Rio de Janeiro, preprint: NF-004/92;

[63] P. P. Srivastava, Higgs mechanism in light-front quantized field theory, December 1991, Ohio-State preprint 92-0012, Contributed to XXVI Intl. Conf. on High Energy Physics, Dallas, TX, August 6-12. AIP Conf. Proceedings No. 272, pg. 2125 (1992), Ed., J.R. Sanford.

[64] P. P. Srivastava, Constraints in light-front quantized theory, Ohio-State preprint 92-0175, AIP Conf. Proceedings No. 272; Nuovo Cimento A107, 549 (1994).

[65] T. Maskawa and K. Yamawaki, Prog. Theor. Phys. 56, 270 (1976).

[66] H. C. Pauli and S. J. Brodsky, Phys. Rev. D 32, 1993 (1985).

[67] Y. Matsumura, N. Sakai, and T. Sakai, Phys. Rev. D 52, 2446 (1995) [arXiv:hep-th/9504150].

[68] J. R. Hiller, S. Pinsky, and U. Trittmann, Phys. Rev. D 64, 105027 (2001) [arXiv:hep-th/0106193].

[69] S. J. Brodsky, J. R. Hiller, and G. McCartor, Phys. Rev. D 64, 114023 (2001) [arXiv:hep-ph/0107038].

[70] S. J. Brodsky, J. R. Hiller, and G. McCartor, Annals Phys. 296, 406 (2002) [arXiv:hep-th/0107246].

[71] S. J. Brodsky, J. R. Hiller, and G. McCartor, arXiv:hepth/0209028.

[72] H. C. Pauli, Nucl. Phys. Proc. Suppl. 90, 154 (2000) [arXiv:hep-ph/0103108].

[73] H. C. Pauli, arXiv:hep-ph/0111040.

[74] T. Frederico, H. C. Pauli, and S. G. Zhou, Phys. Rev. D 66, 116011 (2002) [arXiv:hep-ph/0210234].

[75] G. McCartor, in Proc. of New Nonperturbative Methods and Quantization of the Light Cone, Les Houches, France, 24 Feb - 7 Mar 1997.

[76] W. A. Bardeen, R. B. Pearson, and E. Rabinovici, Phys. Rev. D 21, 1037 (1980).

[77] S. Dalley, Phys. Rev. D 64, 036006 (2001) [arXiv:hep$\mathrm{ph} / 0101318]$.

[78] S. Dalley and B. van de Sande, Phys. Rev. D 59, 065008 (1999) [arXiv:hep-th/9806231].

[79] M. Burkardt and S. K. Seal, Phys. Rev. D 65, 034501 (2002) [arXiv:hep-ph/0102245].

[80] M. B. Hecht, C. D. Roberts, and S. M. Schmidt, nuclth/0008049.

[81] S. J. Brodsky, R. Roskies, and R. Suaya, Phys. Rev. D 8, 4574 (1973).

[82] J. M. Cornwall and J. Papavassiliou, Phys. Rev. D 40, 3474 (1989).

[83] S. J. Brodsky and H. J. Lu, Phys. Rev. D 51, 3652 (1995) [arXiv:hep-ph/9405218]. 